\title{
Derailment von Führungskräften
}

\section{Astrid Schreyögg}

Online publiziert: 12. Mai 2016

(C) Springer Fachmedien Wiesbaden 2016

In den letzten Jahren häufen sich - wahrscheinlich auch durch die Vorkommnisse bei VW motiviert - Berichte über „Derailment von Führungskräften“. Dabei handelt es sich um Entgleisungen unterschiedlicher Art. Manager, seltener Managerinnen, geraten in innere, oft auch in äußere, nicht mehr beherrschbare Zustände von Verwirrtheit. Solche Erscheinungen bedrohen nicht nur die betreffenden Führungskräfte in ihrer individuellen Karriere, sie haben z. T. auch verheerende Auswirkungen für ihre Mitarbeiter - und natürlich auch für die gesamte von ihnen geleitete Organisation oder organisatorische Einheit. Solche Zustände bedürften vielfach externer Hilfen, weshalb es auch für die Berater-Community lohnend ist, sich damit zu befassen. Die Herausgeber von OSC wollten dieser Tatsache Rechnung tragen, weshalb sie hier eine ganze Reihe von Beiträgen zum Thema „,Derailment von Führungskräften“" präsentieren. Hier soll dann das Phänomen aus unterschiedlichen Perspektiven beleuchtet werden.

Im ersten Beitrag führen Rabea Haag und Heidi Möller, Universität Kassel, zunächst in die Thematik mit ihren bekannten Risikofaktoren ein. Sodann differenzieren sie anhand ihrer empirischen Forschung zwei potenzielle Verlaufsformen: Eine Form, bei der die Führungskräfte ,implodieren“, d. h. psychisch erkranken etwa im Sinne von Depression. Andererseits extrahierten die Autorinnen eine Form des „Explodierens“", bei dem die Führungskräfte einen erheblichen Schaden in ihrem Umfeld anrichten. Abschließend werden Empfehlungen für die Diagnostik, die Prävention und die Behandlung ausgesprochen.

Die beiden Wirtschaftswissenschaftler Thomas Kuhn und Jürgen Weibler von der Fernuniversität Hagen diskutieren im nachfolgenden Beitrag Management-Derailment (MD) primär unter dem Gesichtspunkt von Unternehmensethik. Sie argumen-

Dr. A. Schreyögg $(\bowtie)$

Breisgauer Str. 29, 14129 Berlin, Deutschland

E-Mail: info@schreyoegg.de 
tieren, dass die Gewinnsucht in Unternehmen ethisch, oft auch rechtlich fragwürdige Praktiken befördere (z. B. VW). Wenn das Topmanagement moralisch und womöglich auch ökonomisch außer Tritt gerät, kann es nicht ausbleiben, dass auch das untere und mittlere Management durch den permanenten Leistungsdruck moralisch zu entgleisen droht. Dies lässt sich auch als ,systemische Kollusion“, ein kollusionäres Zusammenspiel von verschiedenen Kräften in einem Unternehmen beschreiben.

Was hilft aber nun dauerhaft? Bei empirischen Untersuchungen von Führungsstilen in einem großen Industrieunternehmen hat sich ein transformationeller Führungsstil, der durch eine hohe Dialogbereitschaft seitens der Führungskräfte charakterisiert ist, auch für die Mitarbeiter am positivsten erwiesen. Antonia Sureth, Sebastian Uedelhoven, Jens Nachtwei und Birgit Postler von der Humboldt Universität zu Berlin können auf der Basis faktorenanalytisch gewonnener Ergebnisse die schon von Lewin und seiner Schule behaupteten Effekte nachweisen, wonach ein autoritärer Führungsstil von den Mitarbeiter am negativsten beurteilt wird.

Im nachfolgenden Beitrag thematisiert Herbert Csef von der Universitätsklinik Würzburg Narzissmus als Basis fürs Derailment von Führungskräften. Er macht auf die doppelte Bedeutung narzisstischer Persönlichkeiten fürs Management aufmerksam: Sie scheinen auf der einen Seite für diese Aufgabe besonders prädesdiniert, sie können aber auf der anderen Seite durch genau diesen Narzissmus besonders zerstörerisch wirken. Damit akzentuiert der Autor wie viele andere Psychoanalytiker die besondere Bedeutung des Narzissmus Konzeptes für die Beratung in Organisationen und die Prävention von Derailment.

Horst Kraemer, ein Trauma-Therapeut, bringt nun noch die Neurowissenschaft ins Spiel. Er betont, dass Derailment erst aus einem Zusammenspiel von Neurowissenschaften, Stressforschung und Verhaltensimmunbiologie ausreichend erklärbar wird. Anhand etlicher Beispiele aus seiner Praxis kann er zeigen, wie ein Ressourcenverlust bei Führungskräften ablaufen kann. Er meint, erst wenn die empirische Forschung in das generelle Führungsverständnis integriert wird, können auch effektive Methoden des Stressabbaus auf wissenschaftlicher Basis in Trainings, Coachings usw. integriert werden.

Bernd Völker und Peter Dahms, beide als Militär-Psychologen bei der Bundeswehr tätig, berichten nun gar von einem lebensgefährlichen Derailment, nämlich von Geiselnahmen. Die Autoren betonen, dass solche Ereignisse in der Zivilgesellschaft zum Glück relativ selten, im Militärischen, zumal bei Auslandseinsätzen durchaus häufiger zu beobachten sind. Und für solche Fälle sind Soldat/innen psychologisch vorzubereiten. Im Prinzip sind die Anregungen der Autoren, wie man sich als Betroffener bei Kidnapping zu verhalten hat, auch für jedermann wertvoll; denn immer wieder kommt es auch bei Banküberfällen und vergleichbaren Vorkommnissen zu Geiselnahmen.

In einem Praxisbericht zeigt Michael Jelinek, wie Fach- und Prozessperspektiven in der Beratung verzahnt werden können. Dazu schlägt er die Zusammenarbeit von zwei Beratern vor, die die jeweilige Perspektive als Prozessberater und als Fachberater verkörpern können. Und in einem sehr aktuellen Diskurs setzt sich Mark Staskiewicz, Supervisor und Coach in Graz, wieder mit einem Vorgang auseinander, bei dem manches ,,aus dem Ruder laufen“ kann: mit der Supervision von Freiwilligen in der Flüchtlingshilfe. Hier sah sich der Autor sogar mit tätlichen Angriffen und 
mit Beschimpfungen als „Verräter“ konfrontiert. Dies öffentlich zu machen, ist eine Voraussetzung dafür, in diesen Konfliktfeldern bestehen zu können.

\section{In eigener Sache}

Der Springer Verlag lädt ein zu einem Journal Day Springer - Psychologie 2016. Der Verlag möchte mit den Herausgebern und Redakteuren der im Verlag erscheinenden Zeitschriften in unserem Fachgebiet (OSC - Organisationsberatung Supervision Coaching; CTP - Coaching - Theorie \& Praxis; ZPS - Zeitschrift für Psychodrama und Soziometrie; GIO - Gruppe. Interaktion. Organisation, bis 2015 unter dem Titel Gruppendynamik \& Organisationsberatung erschienen) sowie mit Leser/innen und Autor/innen ins Gespräch kommen, die Konzepte der Zeitschriften diskutieren, Fragen beantworten und Ideen austauschen.

Freitag, 4.11.2016, 11.00-19.00 Uhr

Springer Verlag Berlin, Heidelberger Platz

Die Veranstaltung ist kostenlos.

Verbindliche Anmeldungen bis zum 15.07.2016 direkt an: elke.janosch@ springer.com 